Applied Mathematical Sciences, Vol. 11, 2017, no. 48, 2383 - 2391

HIKARI Ltd, www.m-hikari.com

https://doi.org/10.12988/ams.2017.77242

\title{
Catastrophe Theory and Postmodern General Equilibrium: Some Critical Observations
}

\author{
Pascal Stiefenhofer \\ Brighton Business School \\ University of Brighton \\ Moulsecoomb \\ BN2 4AT, Brighton, United Kingdom
}

Copyright (c) 2017 Pascal Stiefenhofer. This article is distributed under the Creative Commons Attribution License, which permits unrestricted use, distribution, and reproduction in any medium, provided the original work is properly cited.

\begin{abstract}
Postmodern general equilibrium theory is the formulation of the Walrasian model in the context of the mathematics of catastrophe theory. In its formulation the model comes equipped with a mapping called the natural projection. The goal of postmodern general equilibrium theory is to extract economic properties from this mapping. However, we show that the natural projection itself is void of any economic content. This observation alone calls for a reexamination of the assumptions and properties of the postmodern general equilibrium model.
\end{abstract}

Mathematics Subject Classification: 91B02, 91B50

Keywords: Catastrophe Theory, General Equilibrium, Smooth Manifolds

\section{Introduction}

Catastrophe theory discovered by René Thom [18] is a mathematical theory about smooth parameterized functions, which might sometimes exhibit discontinuous behavior (catastrophes) for small changes in the parameters. Thom's theory uses singularities of smooth functions to model nature. Over the recent decades, the theory has not only attracted much attention in academia but also in the media due to its universal character and its applications in 
physics, biology, and social sciences. The mathematically sophisticated theory, however, has not always been applied successfully to model real world phenomena in the social and biological sciences, and therefore, is not without stigma [12],[13],[15],[16].

General equilibrium theory discovered by Leon Walras ${ }^{1}$ is an economic theory aiming at explaining the simultaneous functioning of interrelated markets as a whole. This is in contrast to partial equilibrium which studies properties of isolated markets. As such, the general equilibrium model is characterized by a system of aggregate functions representing the intersection of market demand and market supply. The objective of general equilibrium theory is to study the properties of the solution set of this system of equations. A major contribution of the theory is a set of definitions and assumptions about the economic agents ensuring that the model's solution set is non-empty. The axiomatic approach, which occurred during the modern era of economics, showed existence of equilibria by application of Brower's fixed point theorem in an influential paper by Arrow and Debreu [2]. Among other major achievements of that era are the well known welfare theorems. Subsequent developments in general equilibrium theory, such as the introduction of smooth economies [[10],[11]] marked the end of the axiomatic approach, and therefore, the beginning of a new era, which gave rise to the catastrophe model. The postmodern equilibrium model was born within the context of smooth economies. A distinctive feature of the postmodern general equilibrium model is that it comes equipped with a natural projection [4]. This puts the economic model in the realm of catastrophe theory. It has been pointed out by various authors that catastrophe theory has not always been applied rigorously in economics. This paper, therefore, aims at testing the mathematical conditions against the economic phenomenon to be studied. First, various issues are pointed out in this paper. These are related to misguiding nomenclature, confusing graphical representations, and the basic assumptions of the model. Second, this paper provides some recommendations about how to overcome some of these difficulties and discusses the limitations of the model.

The organization of the paper is as follows: The next session discusses the core mathematical building blocks of applied elementary catastrophe theory. Section three introduces and example of a postmodern general equilibrium model with its assumptions. Section four presents the main results, observations, and their discussion. The final section is a conclusion. It also provides suggestions for further research.

\footnotetext{
${ }^{1}$ Elements of Pure Economics: English translation of its original in (1874), Corbaz, Lausanne.
} 


\section{Applied Elementary Catastrophe Theory}

Elementary catastrophe theory considers a smooth parameterized real valued potential function, which, depends on two or less state variables, and four or less control parameters [8],[12],[14]. The objective of the potential function is to determine the modeling conditions of real world phenomena. In the context of this paper, elementary catastrophe theory deals with the study of singularities and the structural stability of the potential function. In particular the theory provides a full classification of degenerate singularities in terms of a finite number of geometric structures. In fact Thom showed that there are only seven generic geometric structures associated with this modeling set up [17]. We now consider the theory of elementary catastrophes from and applied perspective.

Let's consider the indispensable core mathematical requirements of applied elementary catastrophe theory when applied to real world problems [12]. At the very heart of elementary catastrophe theory there is a potential function

$$
V: X \times U \rightarrow \mathbb{R}
$$

where $X \subset \mathbb{R}^{n}$ is called the state space, and $U \subset \mathbb{R}^{r}$ is called the parameter space. The objective of this function is to model some physical phenomena of interest. The stable states of this system are regarded as those obtained by minimizing the potential function. If multiple minima exist, then more then one stable state is permitted by the system. A smooth change in the parameter space $u \in U$ will alter the governing potential inunction $V$ in various ways. In fact the stable states of the system may change in a discontinuous manner. This abrupt change is called "catastrophe". The potential function $V$ can also be represented as a smooth family of parameterized potential functions

$$
V_{u}(x): \mathbb{R}^{n} \rightarrow \mathbb{R}
$$

where $u \in U$. The second core mathematical ingredient of elementary catastrophe theory is a set of critical values

$$
M_{V}:=\left\{(x, u) \in \mathbb{R}^{n} \times \mathbb{R}^{r}: \nabla_{x} V(x, u)=0\right\} .
$$

In many application, $M_{V}$ can be shown to be a smooth manifold of dimension $r$. This however, is not always obvious and may require a proof. This Manifold is generated by imposing a first order condition on the potential function. The points on the manifold $M_{V}$ at which the tangent plane is vertical corresponds to the critical points which are degenerate denoted by $\mathcal{C}$. These points can be obtained by solving the system of equations given by $\nabla V_{x}=0$ and $\nabla^{2} V_{x}=$ 0 . The points on $M$ at which the tangent plane is vertical are considered here to be a smooth manifold $\mathcal{C}$. In general $\mathcal{C} \subset M_{V}$. 
The final indispensable mathematical requirement of applied elementary catastrophe theory is the existence of a catastrophe mapping

$$
\mathcal{X}_{V}: M_{V} \rightarrow \mathbb{R}^{r}
$$

induced by the natural projection $\mathcal{X}: \mathbb{R}^{n+r} \rightarrow \mathbb{R}^{r}$. This mapping is called the catastrophe map of $V$. The catastrophe map is the restriction of the natural projection $\mathbb{R}^{n+r} \rightarrow \mathbb{R}^{r}$ to $M_{V}$. The set of critical points $\mathcal{C}$ is the set of critical points in $M_{V}$ of the catastrophe map $\mathcal{X}_{V}$, at which $\mathcal{X}_{V}$ is critical, that is, the rank of the partial derivatives $D_{x} \mathcal{X}_{V}$ is less than $r$. The image of $\mathcal{C}$ under the catastrophe map $\mathcal{X}_{V}$ yields the bifurcation manifold $\mathcal{B}$. The central object of study of applied elementary catastrophe theory is the analysis of the properties of the natural projection and its restriction to the the set of critical values of $V$ denoted by manifold $M_{V}$.

\section{A Pure Exchange Economy}

We impose only minimal assumptions on the general equilibrium model. In fact, we consider the simpliest Arrow-Debreu general equilibrium model possible [9]. Hence, let's consider a pure exchange economy with 2 consumers indexed by $i=1,2$. There are two commodities $x_{i}$ indexed by $k=1,2$. Hence a commodity bundle is a vector $x_{i}=\left(x_{i}^{1}, x_{i}^{2}\right)$. We define the consumption space to be the strictly positive orthant by

$$
X=\mathbb{R}_{++}^{2}:=\left\{x_{i} \in \mathbb{R}^{2}: x_{i}^{k}>0 \text { for } k=1,2\right\} .
$$

Associated with a commodity bundle is a strictly positive price vector $p=$ $\left(p^{1}, p^{2}\right)$. We choose the numeraire price $p^{2}=1$. The set of numeraire normalized prices is defined by

$$
\mathcal{S}:=\left\{p \in \mathbb{R}_{++}^{2}: p^{2}=1\right\} .
$$

We now consider the space of initial endowments. Each consumer $i$ is endowed with a commodity bundle $\omega_{i} \in X$, where $\omega_{i}=\left(\omega_{1}^{1}, \omega_{i}^{2}\right)$. The economy is parameterized by $\omega_{i}$ with parameter space

$$
\Omega:=X^{2} \subseteq\left(\mathbb{R}_{++}^{2}\right)^{2} .
$$

The economic space of our model is defined by

$$
\mathcal{S} \times \Omega
$$

Let consumer $i$ 's preferences be represented by a surjective and differentiable $\operatorname{map}[10]$

$$
u_{i}: X \rightarrow \mathbb{R}
$$


The model of the consumer is then to maximize utility subject to a wealth constraint given by

$$
\left(x_{i}\right) \in \operatorname{argmax} u_{i}\left(x_{i}\right): p \cdot x_{i} \leq w_{i},
$$

where the consumer's wealth $w_{i}$ is defined by $w_{i}:=p \cdot \omega_{i}$. The solution to the consumer's problem is a smooth demand function

$$
f_{i}: \mathcal{S} \times \mathbb{R}_{++} \rightarrow \mathbb{R}_{++}^{2}
$$

satisfying Walra's law

$$
p \cdot f_{i}\left(p, w_{i}\right)=w_{i}
$$

We are now in a position to formulate the equilibrium equation of this pure exchange economy. Let

$$
Z: \mathcal{S} \times \Omega \rightarrow \mathbb{R}
$$

defined by

$$
z(p ; \omega):=\sum_{i}\left(f_{i}\left(p, p \cdot \omega_{i}\right)-\omega_{i}\right)=0 .
$$

It states that for every $\omega \in \Omega$ aggregate demand equals aggregate supply. The solution to the equilibrium equation is a set of pairs $(p, \omega)$ in $\mathcal{S} \times \Omega$ denoted by $E$. The equilibrium equation satisfies Walra's identity $p \cdot z(p, \omega)=0$.

The equilibrium manifold can be show to be a smooth manifold

$$
E:=\{(p, \omega) \in \mathcal{S} \times \Omega: z(p ; \omega)=0\}
$$

of dimension 4. This number can easily be calculated by application of the regular value theorem to the equilibrium equation. Hence, locally the structure of the equilibrium manifold $E$ is that of the Euclidean space $\mathbb{R}^{4}$. The nonemptiness of $E$ was shown by Arrow and Debreu [2].

Assumption 1 ([4]) Let there exists a mapping

$$
\pi: E \rightarrow \Omega
$$

where $E$ is a smooth manifold. This mapping is called the natural projection.

The natural projection is a mapping from points $(p, \omega)$ in $S \times \Omega$ restricted to $E$ into $\Omega$. The general equilibrium model above together with assumption 1 defines the postmodern general equilibrium model. 


\section{Results and observations}

Section 3 formulates the pure exchange general equilibrium model in the language of catastrophe theory. In this formulation, the Arrow-Debreu model comes equipped with a natural projection, a mapping from the set of solutions of the model into the parameter space [5]. We assume only minimal conditions on the number of goods and the number of consumers that satisfy the requirements of a general equilibrium model. This section examines the economic and mathematical conditions of the model above in the context of applied elementary catastrophe theory.

Observation 1 The first mathematical condition of elementary catastrophe theory is the existence of a minimizing potential function (1). A potential function is a real valued function describing the phenomena of interest. In essence, it directly represents the model of interest to the researcher.

The general equilibrium model considered in section three does not meet the mathematical requirement regarding existence of a potential function. (i) The equation (4) given by $z(p ; \omega)=0$ is an economic equilibrium condition imposed on the model. It does not describe the inner working of the economic model itself. For example, the behavior of the economic agents is not deduced from this equation. (ii) From the same equation (4) we learn that, even in the simplest formulation of the general equilibrium model considered in section three, it is a vector valued function for which a minimizing (or maximizing) criterion does not exist.

Observation 2 The set of critical points $(x, u) \in X \times U$ is provided by definition (2). By definition, this set contains all points $(x, u) \in \mathbb{R}^{n} \times \mathbb{R}^{r}$ satisfying the condition $\nabla_{x} V(x, u)=0$.

The construction of the two sets $E$ and $M_{V}$ are fundamentally different in nature. By construction, the equilibrium manifold $E$ consists of the points $(p, \omega) \in S \times \Omega$ that are solutions to the system of equations (4), while the later is the set of singularities (minima) of a real valued function representing the physical phenomena of interest. The set $E$ does not represent a set of singular points as it is typical in catastrophe theory. This observation follows from the lack of existence of a minimizing potential function describing the economic model. Moreover, the conditions for classification of degenerate critical points $\nabla V_{x}=0$ and $\nabla^{2} V_{x}=0$ ( and higher order derivatives) do not apply to the the general equilibrium model. However, it is precisely this classification of singularities that is of central object of study in applied elementary catastrophe theory.

Observation 3 The dimension of $M_{V}$ is $r$. 
Balasko [[3], [4]] shows that the set of equilibria $E$ is a smooth manifold of dimension $m l$, where $m$ is the number of consumers and $l$ is the number of commodities. He postulates that it is precisely this general result that motivates the application of differential topology and catastrophe theory in the context of the general equilibrium model. By inspection of the general equilibrium model in section three where $m=2$ and $l=2$, it follows that the $\operatorname{dim}(E)=4$, which, corresponds to the dimension of the parameter space $\Omega$ of the model. The generality of this result, however, is in contrast to many graphical representations of the equilibrium manifold. Such illustrations appear i.e., in Balasko ([6],p.33,53,66), (Balasko [7],p.18,21) Mas-Collel ([1],p.596),

In this paper we propose a result, which is independent of the number of economic agents. The result permits the graphs illustrated in above references. Our result however, requires a reformulation of the aggregate commodity space, which is independent of the consumer index $i=1, \ldots, n$.

Theorem 1 The set of equilibria $\mathcal{E}$ is a smooth submanifold of $\mathbb{S} \times \Omega$ of $d i$ mension $l$.

Proof. Let $X_{i} \subset \mathbb{R}^{l}$ be the consumption space of consumer $i$. Let the aggregate consumption space be defined by $\sum_{i} X_{i}=X$ which satisfies $X \subset \mathbb{R}^{l}$. Similarly, let $\Omega_{i} \subset \mathbb{R}^{l}$ be the space of initial endowments for consumer $i$. Let the aggregate the aggregate endowments pace be defined by $\sum_{i} \Omega_{i}=\Omega$ which satisfies $\Omega \subset \mathbb{R}^{l}$. By Walra's law and the equilibrium equation $Z$ it follows that there are $l-1$ independent coordinates on $\hat{Z}$. The set $\mathcal{E}$ is the preimage of $0 \in \mathbb{R}^{l-1}$ defined by the map $\hat{Z}: \mathcal{S} \times \Omega \rightarrow \mathbb{R}^{l-1}$ such that $Z(p, \omega)=0$ is satisfied. We now calculate the Jacobian matrix of $Z(p, \omega) w . r . t$. the endowments $\omega$. This yields after a simple calculation and some algebraic manipulation

$$
J_{\omega}=\left(\begin{array}{ccccc}
-1 & 0 & 0 & 0 & \frac{\partial f^{1}}{\partial w} \\
0 & -1 & 0 & 0 & \frac{\partial f^{2}}{\partial w} \\
\vdots & \vdots & \ddots & \vdots & \vdots \\
0 & 0 & 0 & -1 & \frac{\partial f^{l-1}}{\partial w}
\end{array}\right)
$$

where $p \cdot \omega_{i}=w_{i}$ is the individual's wealth and $f^{k}$ is the aggregate demand function for commodity $k \in 1, \ldots, l$. By computation, the rank of $J_{\omega}$ is $l-1$ by equation (7). Hence by the regular value theorem $0 \in \mathbb{R}^{l-1}$ is a regular value of $\hat{Z}$. Moreover, the regular value theorem also tells us that the dimension of $E$ is $l-1+l-(l-1)=l$. This completes the proof.

Observation 4 The natural projection is the map $\mathcal{X}: \mathbb{R}^{n+r} \rightarrow \mathbb{R}^{r}$. Its restriction to the set $M_{V}$ is the catastrophe map $\mathcal{X}_{V}: M_{V} \rightarrow \mathbb{R}^{r}$. 
There seems to be a confusion of terminology in the general equilibrium literature regarding mathematical nomenclature. In the context of general equilibrium, the natural projection is a map $\pi: E \rightarrow \Omega$ by definition 3. This map is the restriction of $\mathbb{R}^{(l-1)+(m l)} \rightarrow \mathbb{R}^{m l}$ to points $(p, \omega)$ in the equilibrium manifold $E$. This terminology is at variance to elementary catastrophe theory in which the same map is called the catastrophe map. The natural projection, sometimes called projection and serves a different purpose. Assumption 1 introduces a smooth map between manifolds. This map does not alter the economic model in any way nor does it provide any further economic insight.

\section{Conclusion}

This paper reformulates the commodity space of the postmodern general equilibrium model and shows that under a set of minimal assumptions the dimension of its solution set corresponds to the dimension of the set of singularities of elementary catastrophe theory. This result rehabilitates the condition stated in observation 3. However, the paper fails to provide an economic justification for the introduction of the catastrophe map. It remains that the catastrophe map of the postmodern general equilibrium model remains an artifact of the mathematical method employed. This follows from observation 2 and assumption 1. A suggestion for improvement on observation 4 was provided.

Based on observation 1, the main conclusion of this paper however, is that a potential function describing the inner workings of the economic model does not exist, suggesting that elementary catastrophe theory seems not to be the appropriate mathematical method of model inquisition. The investigation whether the economic model can be reformulated in the context of a potential function remains an open problem.

\section{References}

[1] M.D. Whinston, A. Mas-Colell and J.R. Green, Microeconomic Theory, Oxford University Press, Berlin, New York, Oxford, 1995.

[2] K. Arrow and G. Debreu, Existence of an equilibrium for a competitive economy, Econometrica, 22 (1954), 265-290.

https://doi.org/10.2307/1907353

[3] Y. Balasko, Some results on uniqueness and on stability of equilibrium in general equilibrium theory, Journal of Mathematical Economics, 2 (1975), 95-118. https://doi.org/10.1016/0304-4068(75)90017-8 
[4] Y. Balasko, Economic equilibrium and catastrophe theory: An introduction, Econometrica, 46 (1978), 557-569. https://doi.org/10.2307/1914231

[5] Y. Balasko, Equilibrium analysis and envelope theory, Journal of Mathematical Economics, 5 (1978), 153-172.

https://doi.org/10.1016/0304-4068(78)90021-6

[6] Y. Balasko, The Equilibrium Manifold: Postmodern Developments in the Theory of General Economic Equilibrium, The MIT Press, Cambridge, Massachusetts, 1988.

[7] Y. Balasko, Foundations of the Theory of General Equilibrium, Academic Press, Orlando, Florida, 1988.

[8] D. Castrigiano and S. Hayes, Catastrophe Theory, ABP, Westview, 2004.

[9] G. Debreu, Theory of Value, Wiley, New York, 1959.

[10] G. Debreu, Smooth preferences, Econometrica, 40 (1972), 603-615. https://doi.org/10.2307/1912956

[11] G. Debreu, Regular differentiable economies, The American Economic Review, 66 (1976), 280-287.

[12] M. Golubitsky, An introduction to catastrophe theory and its applications, SIAM Review, 20 (1978), no. 2, 352-387. https://doi.org/10.1137/1020043

[13] J. Guckenheimer, Comments on Catastrophe Theory and Chaos, Preprint, 1978.

[14] D. Postle, Catastrophe Theory, Fontana, 1980.

[15] S. Smale, Stability of Unfoldings, Springer, New York, 1980.

[16] H.J. Sussman and R.S. Zahler, Catastrophe theory as applied to the social and biological sciences: A critique, Synthese, 37 (1978), 117-216. https://doi.org/10.1007/bf00869575

[17] R. Thom, Les singularites des applications differentiables, Annales Institute Fourier, 6 (1956), 43-87. https://doi.org/10.5802/aif.60

[18] R. Thom, Structural Stability and Morphogenesis, Benjamin-AddisonWesley, 1975.

\section{Received: August 15, 2017; Published: September 22, 2017}

